\title{
Retraction: Blackbody friction force on a relativistic small neutral particle [Phys. Rev. A 91, 032505 (2015)]
}

\author{
A. I. Volokitin
}

(Q) (Received 23 December 2019; published 3 January 2020)

DOI: 10.1103/PhysRevA.101.019901

Significant portions of this article were previously published as A. I. Volokitin, Friction force at the motion of a small relativistic neutral particle with respect to blackbody radiation, Pis'ma Zh. Eksp. Teor. Fiz. 101, 479 (2015) [JETP Lett. 101, 427 (2015)], on 9 February 2015. In light of this overlap, this article is considered retracted. 